\title{
The Effect of Coupled Mass Transport and Internal Reforming on Modeling of Solid Oxide Fuel Cells Part II: Benchmarking Transient Response and Dynamic Model Fidelity Assessment
}

\author{
K. J. Albrecht, R. J. Braun* \\ Department of Mechanical Engineering, College of Engineering and Computational Sciences, Colorado \\ School of Mines, 1610 Illinois Street, Golden, CO 80401, USA
}

\begin{abstract}
One- and 'quasi' two-dimensional (2-D) dynamic, interface charge transport models of a solid oxide fuel cell (SOFC) developed previously in a companion paper, are benchmarked against other models and simulated to evaluate the effects of coupled transport and chemistry. Because the reforming reaction can distort the concentration profiles of the species within the anode, a 'quasi' 2-D model that captures porous media mass transport and electrochemistry is required. The impact of a change in concentration at the triple-phase boundary is twofold wherein the local Nernst potential and anode exchange current densities are influenced, thereby altering the current density and temperature distributions of the cell. Thus, the dynamic response of the cell models are compared, and benchmarked against previous channel-level models to gauge the relative importance of capturing in-situ reforming phenomena on cell performance. Simulation results indicate differences in the transient electrochemical response for a step in current density where the 'quasi' 2-D model predicts a slower rise and fall in cell potential due to the additional volume of the porous media and mass transport dynamics. Delays in fuel flow rate are shown to increase the difference observed in the
\end{abstract}

\footnotetext{
${ }^{*}$ Corresponding author. Tel.: + 303273 3055; fax: + 3032733602 .

Email addresses: kalbrech@mymail . mines . edu (K. J. Albrecht), rbraun@mines . edu (R. J. Braun
} ) 
electrochemical response of the cells.

Keywords: Dynamic, SOFC, Modeling, Mass Transfer, Dusty Gas Model, Internal

Reforming

\section{Introduction}

Solid oxide fuel cells (SOFC) have received continued interest over the past decade due to their high electrical efficiency, fuel flexibility, and high quality waste heat. These benefits suggest that SOFCs are likely to play a role as a distributed energy resource, especially if life cycle cost can be reduced or significant incentives such as a carbon tax are implemented. At the current point in SOFC development, degradation effects severely limit the operational lifetime of SOFCs. The development of models which can determine when harmful operational conditions maybe encountered are paramount to commercial success. Although the focus of this paper is not on quantifying degradation effects, they are considered when assessing the level of fidelity necessary for accurately predicting cell performance.

Proper selection of SOFC model fidelity is typically guided by model purpose but is often challenged by a lack of understanding of the implications of various model simplifications that are usually made for the purposes of computational efficiency and the availability of experimental data to support model validation. Dynamic, interface charge transport channel-level modeling requires the implementation of transient species, energy, and momentum balances coupled with additional submodels to capture the porous media mass transport and electrochemistry of the cell. Many channel-level models implement the same modeling methodology of considering the diffusion within the porous anode as both a linear reduction in hydrogen and a linear increase in steam concentration, which are proportional to the local current density. This assumption is made to decouple the effects of the reforming reaction on the electrochemical model, allowing for the formulation of a one-dimensional (1-D) model. In cells where internal reforming of methane occurs, this assumption is violated due the diffusion of 
methane into the porous anode where the steam reforming reaction consumes water and produces hydrogen, which is catalyzed by the nickel surface. The reforming reaction distorts concentration profiles of the species within the anode, where hydrogen concentration at the TPB may be higher or lower than that of the fuel channel depending on the operating conditions and position along the length of the cell. Thus, because of local concentration variations within the cell layers, transport resistances and electrochemical performance may be quite different than in either hydrogen-only supplied SOFCs or during the relatively short time intervals when the cell is responding to dynamic load change. The present work is a companion paper to a prior model development effort [4], which seeks to evaluate model fidelity when simulating the transient response of SOFCs, thereby elucidating the circumstances which justify higher-order modeling efforts intended to support system-level SOFC performance modeling.

Over the past years there have been countless modeling efforts of dynamic SOFCs with system-level simulations in mind [2, 1, 3, 9, 11, 13, 15, 19, 20, 21, 14], including both lumped and spatially resolved models. Typically, these models include oversimplifications of the physical processes occurring in the cell such as internal reforming, mass transport, and electrochemical kinetics. The reason behind the simplification is to decouple the effects of electrochemistry and internal reforming, leading to a more computationally efficient model, where the cell is approximated as lumped or over a single dimension. However, neglecting the coupled effects could influence cell behavior and operational envelopes. When the goal of the model is to assess potentially harmful system transient effects on the cell and other balance-of-plant (BOP) components, a model that is physically based and verified against experimental data is paramount.

\section{Prior SOFC Dynamic Modeling \& Verification}

Our previous work [4] has developed and discussed the multi-dimensional, interface charge transfer channel-level models for both steady-state and dynamic operation. 
Experimental verification of dynamic fuel cell models is quite difficult to accomplish in the laboratory, as it requires fast, time dependent data for cell temperature, voltage, and gas species evolution, including streamwise profiles of locally measured variables. Additionally, labscale verification against a cell within a furnace is not entirely representative of a adiabatic cell within a system. Lacking such data, the approach taken here [20] is to benchmark our results against other models in the extant literature. Thus, this paper focuses specifically on channel-level dynamic models and a direct comparison of the levels of fidelity implemented. Prior to presenting benchmarking results, we first give a brief overview of prior relevant dynamic modeling work.

Dynamic channel-level SOFC models can be divided into varying levels of fidelity, which are dependent on the level of spatial discretization. The simplest dynamic model is a lumped model where no spatial discretization is present, these models are computationally efficient and a good option for model predictive control or where rapid dynamic simulation is of interest [23, 17]. The term lumped has also been used to classify the resolution of different layers within a spatially distributed model where the temperature has been lumped transverse to the bulk flow resulting in a model which falls in between the fully lumped model and the 1-D channel-level model [12]. Theses models have merit from a system-level modeling perspective, however, effects of flow configuration and temperature distribution are lost due to the lumped nature.

The 1-D model of intermediate fidelity is highly utilized in dynamic modeling efforts particularly on the system-level where the temperatures for different layers have been resolved transverse to the axial flow, however, the temperature or species gradients within the specific layers have not been resolved transverse to the axial flow $[2,3,11]$. Transport between the different layers is established based on a resistive network where convection coefficients based on Nusselt number correlations and plug flow have been assumed. This methodology is capable of simulating a counter or coflow configuration, however, species gradients within the anode and the anode volume 
contributing to species transients have been neglected.

The 'quasi' 2-D model of higher fidelity extends the 1-D methodology and resolves the species profile in a second dimension throughout the depth of the anode $[8,9]$. However, the channel composition and temperature are still considered to be lumped. This model extends the 1-D dynamics and is able to capture the species transients within the anode, and the effect of the internal reforming on the current density and temperature profile of the cell. The species transients are important to capture in SOFC modeling since prior system-level modeling results have indicated that dynamic loadfollowing ability is limited by anode fuel depletion. Additionally, this model allows for a robust simulation tool where the electrode design can be easily modified to determine the dynamic and steady-state operational benefits.

High fidelity 2-D or 3-D models have been developed as well, where the species and temperature distributions have been fully resolved in space $[7,5]$. This modeling methodology has been implemented from button cells and simplified geometries to the full cell level models. The dynamics of these model suggest that they would be difficult to implement into a system-level model. However, dynamic system-level models with this level of fidelity still have been developed [18]. These models provide useful results for known geometries or commercial designs, however, system configuration studies or general results would be difficult to obtain where exact geometries are unknown. Simulation of 3-D geometries would require extensive knowledge of the stack/system including sealing, stack manifolding, hardware packaging details which describe both the relative location of the individual system components, and insulation characteristics, and detailed dimensional information of the system - all of which are typically closely guarded as proprietary.

The development of design and simulation tools, which are computationally efficient and capture the coupled effects of parameter changes on the cell performance, are still important to develop. Many dynamic SOFC models have been developed to 
assess the effects of transient SOFC operation on performance. An in-depth review of these models is provided in our companion paper to the present work[4] . Here we emphasize that one of the main differences between prior efforts and the present work is the assessment of the importance of model fidelity as it pertains to resolving electrode species transport and reaction during steady-state operation. In particular, we establish the importance of detailed reaction and transport modeling (via resolving the anode electrode) on cell-level model-prediction whose primary purpose is implementation in stack and system-level simulation. The remaining sections of this paper will benchmark the dynamic response of the current models against previous modeling efforts, and compare the dynamic operation of the two models developed in the companion paper by the authors [4].

\section{Dynamic Channel-Level Benchmarking}

In order to verify that the modeling results are consistent with prior modeling efforts, the model has been benchmarked against the electrochemical response of other models $[10,1,22,11,20]$ for a step change in voltage, current density or resistance. The model was operated in co-flow configuration for a step change in current density from 3000 to $5000 \mathrm{~A} \mathrm{~m}^{-2}$. The transient cell potential has been scaled as done by Qi et al. [20] and compared to the other modeling results (Figure 1) as compiled by Qi et al. [20]. The time scale of the dynamic response of the model is shown to be in agreement with previous results. Exact agreement between models in not expected since the solutions presented will be dependent on modeling parameters, geometry, flow configuration, and control strategy (Table 1). The current model agrees well with the most recent modeling efforts of Qi et al. The differences between the modeling results can be attributed to model geometry (tubular or planar) or control strategy (constant fuel utilization or flow rate). However, the qualitative comparison of the results indicates that the general characteristic of the dynamic response is in agreement with the prior 
results where a large drop in cell potential followed by a slow rise to the steady-sate value is observed.

Experimental data for model validation is limited in the thermal time scale of 1000 seconds, but experimental data and model validation for small time scales of 1-2 seconds have been completed by Bhattacharyya et al. [6]. However, little information was given about the tubular cell operating conditions, and comparisons for the current model were unable to be completed. Additionally, modeling in this specific time scale could be influenced by other unmodeled effects including the capacitance of the double layer, which is not captured here.

\section{Dynamic Channel-Level Modeling Comparison}

Simulations have been conducted for cell response to a step in current density where the inlet fuel composition and the utilization of the cell and air ratio were held constant. The operating parameters are given in Table 2. This operating strategy is difficult to accomplish in practice due to blower or valve dynamics not being infinitely fast, however, the purpose here is to isolate the cell-level dynamics from the balance-ofplant. The current density step responses were simulated for a 0.001 second ramp in current density from the initial to final value. The differences in transient response of the maximum PEN temperature, PEN temperature gradient, cell potential, and cathode outlet temperature were investigated between the two models. The results have been reported for both counter and co-flow configurations to reveal if dynamic operation results are flow configuration dependent.

\subsection{Co-Flow Configuration}

The co-flow configuration response for cell voltage to both a step up and down in current density is displayed in Figure 2. The model is initialized for steady-state con-

ditions at a specified current density and simulated for 100 seconds where the step is imposed on the model. The step up in current density displays a large overshoot in cell 
voltage where the operating voltage temporarily falls below the steady-state value and eventually returns. The step down in current density also displays an overshoot with the voltage rising sharply above its steady-state value followed by a sharp drop below before asymptotically reaching steady-state. The dynamic response of the voltage can be attributed to the dynamics of the species in the fuel channel and the thermal response of the PEN temperature. The step up in current density initially results in the voltage falling below the steady-state value since the fuel species in the channel become depleted before the anode fuel species distribution is able to reach the new steady-state value. The slower dynamic is due to the nominal PEN temperature rising to the new steady-state value reducing overpotetnials and increasing the operating voltage. The step down in current density initially results in an increase in the cell voltage past the steady-state value due to a surplus of species in the anode channel before the fuel species distribution have reached the new steady-state values. The voltage then falls below the steady-state value due to the dynamics of the PEN temperature distribution. Overall, both of the models predict similar steady-state and dynamic voltage results that are independent of modeling methodology. Only the small differences reported in the steady-state model are displayed in the dynamic response, except for the dynamics around the step.

The reduction in cell voltage following the step change in current density was completed through holding the fuel utilization constant where the inlet flow rate of the fuel is directly proportional to the current of the cell. However, the fuel utilization will be a dynamic quantity since the step in current density will deplete the concentration of fuel along the length of the channel. This definition of fuel utilization is related to the potential drop that is experienced by the cell following the step increase in current density since reversible cell voltage is concentration dependent. For increases in current density the fuel species will become depleted and utilization of the fuel will temporarily rise where step decreases in current density will result in excess fuel present in the fuel 
channel resulting in a temporary decrease in fuel utilization. Differences between the 1-D and 'quasi' 2-D models are observed since, for the anode supported cell, less fuel depletion will occur in the 'quasi' 2-D model since the volume of the anode contains additional fuel, which is not modeled in the 1-D case.

In addition to the electrochemical dynamic response, the thermal response of the air leaving the cell is important to accurately capture. Since the cathode gas stream has a significantly higher thermal capacitance than the fuel stream, the dynamic response will govern the response of the down stream BOP components (combustor, pre-reformer, heat exchangers). The modeling comparison for the cathode outlet temperature dynamic (not shown) displays slight differences in the outlet temperature dynamic response, however, the differences are minimal and will likely be unimportant in system-level modeling. The characteristic of the dynamic response shows large temperature changes at the step. These changes are due to the relatively low thermal capacitance of the gas phase species compared to the solid PEN structure. For the step down in current density, the temperature of the cathode gas at the outlet is less than the PEN temperature initially. During the step, the flow rate of the gas is reduced and the residence time is increased allowing the temperature of the gas stream to more closely reach that of the PEN. For the step up in current density, the abrupt decrease in cathode outlet temperature is due to the opposite effect. A rapid change in temperature after the current step up is observed before the cathode outlet temperature settles to the steady-state value, registering only minimal overshoot in the process.

The dynamics of the evolving PEN temperature distribution and resulting thermal stress internal to the cell are important to capture in dynamic modeling. The maximum PEN temperature and temperature gradient have been previously identified as potential variables associated probability of failure due to thermal stress [16] where accurate knowledge of their values during system-level supervisory control and simulation is imperative. The maximum PEN temperature response for both modeling methodolo- 
gies is displayed in Figure 3 and the maximum PEN temperature gradient is displayed in Figure 4. The dynamic response for both variables is not significantly dependent on the methodology used or the directionality of the step for the case of the co-flow configuration.

A comparison of the modeling methodology on channel-level dynamics in co-flow configuration indicates that the dynamic response of the variables of interest are not significantly altered by the modeling methodology implemented. As with the steadystate model analysis, a 1-D model is sufficient for capturing the co-flow channel-level model dynamics. The dynamic response of a 'quasi' 2-D model will only differ from the 1-D case when the time scale of interest is affected by the porous media mass transport dynamic, which are not captured by the 1-D model.

\subsection{Counter-Flow Configuration}

Repeating the comparison for a counter-flow configuration cell results in the same steady-state differences that were observed and reported previously in the companion paper [4], namely that maximum cell temperature and maximum cell temperature gradient increase with decreased pre-reforming and increased current density.

The electrochemical performance of the cell displays a dynamic response (Figure 5) similar to the co-flow configuration, however, the steady-state electrochemical performance of the models have been shown to contain differences. These differences affect the steady-state voltage predictions and the dynamic values, however, the characteristic of the dynamic response is similar. The 1-D model predicts more fuel depletion and a larger change in the transient fuel utilization quantity due to the step change in current density compared to that of the 'quasi' 2-D model.

The dynamic cathode gas outlet temperature response (not shown) indicates the steady-state modeling predictions of cathode outlet temperature are almost identical, however, the dynamic response is affected by the modeling methodology. The magnitude of the temperature change after the step response and the dynamics of the outlet 
temperature prior to reaching the steady-state value both display differences. For the step down in current density, the 'quasi' 2-D model predicts a smaller change in the cathode outlet temperature. Since the reforming occurs over the entrance region of the fuel channel, which is adjacent to the outlet of the air channel, the high reforming rate predicted by the 1-D model will result in larger drops and increases in the cathode outlet temperature. The approach to modeling the fuel reforming process will affect the cathode outlet temperature dynamics, however, the temperature differences are only found to be in the range of 3-4 K.

The dynamic response of the cell temperature gradient within the counter-flow cell demonstrates that there are additional differences simulation results depending on the modeling methodology. Both steady-state and dynamic differences in modeling approach are observed for the dynamic response of the maximum temperature experienced by the PEN layer (Figure 6). The step up in current density will move the maximum PEN temperature in different directions after the step has been completed. This is caused by the reduced reforming rate of the 'quasi' 2-D model and increase in fuel flow rate. Since the reforming rate of the 'quasi' 2-D model is less than that of the 1-D model, the step response allows for the fuel reforming to take place over a larger length of the cell, ultimately reducing the maximum PEN temperature. For the step down in current density, the dynamic response displays similar characteristics, however, the values are offset by the steady-state difference. The dynamic response of the maximum PEN temperature gradient is displayed in Figure 7, where the 1-D model predicts significantly higher PEN temperature gradients in steady-state operation. Additionally, the maximum PEN temperature gradient dynamic contains larger overshoot for the 1-D model. Since the 1-D model has a higher methane reforming rate over the fuel inlet as previously discussed, the temperature gradient will more rapidly approach the new steady-state value, although the settling times are comparable. 


\section{Mass Transport Dynamics}

The mass transport dynamics included in the 'quasi' 2-D model, but not the 1-D model, were shown to affect the model response in the one second time scale following the step in current density. In order to isolate the effect of the mass transport dynamics on a cell-level model simulations, results that include and neglect mass transport dynamics are presented for a 'quasi' 2-D model where both models will reach the same steady-state values.

The comparison of the responses for both co- and counter-flow configurations are presented in Figure 8 for step increases and decreases in current density. The modeling comparison displays small difference in the voltage for a perfectly controlled cell where the air ratio and fuel utilization are held constant. However, blower or compressor dynamics will delay the fuel and air flow rate in reality. Simulation for a 0.15 s delay in fuel and air flow rate are presented as well where the dynamic voltage significantly differs between models when the mass transport dynamics are neglected or included. When the mass transport dynamics are included, the model accounts for the fuel stored in the channels as well as the anode support layer. The additional fuel modeled with the mass transport dynamics results in less severe changes in cell voltage when the current density is stepped due to the additional fuel volume modeled in the anode support. Neglecting the mass transport dynamics and subsequently the fuel stored in the anode support volume, results in more significant changes in cell voltage especially in the case where fuel and air flow delays are considered. However, the nature of the differences reported here will depend on the exact geometry of the anode (i.e., thickness, porosity, and tortuosity). The simulation results presented are for parameters typical of a planar anode-supported SOFC geometry; the modeling differences would be less for an electrolyte-supported cell. 


\section{Radiation Boundary Condition}

Radiation heat loss from the stack can be accounted for by altering the boundary conditions for the conduction of the PEN and interconnect. Performing an energy balance on the end nodes where the conduction will be equal to the radiation heat loss gives the expression in Equation 1 for the temperature gradient at the PEN and interconnect boundaries. The values for the emissivity $(\varepsilon)$ have been taken from the work of Aguiar et al. [2] and Qi et al. [20]where the PEN and interconnect have been set to values of 0.8 and 0.4 , respectively. The case temperature has been varied to determine the sensitivity of the solution to this parameter.

$$
\frac{\partial T}{\partial x}=\frac{\varepsilon \sigma}{k}\left(T_{\text {case }}^{4}-T^{4}\right)
$$

The simulation results for the PEN and interconnect temperature distribution and current density are displayed in Figure 9. Results are presented for a counter-flow configuration cell where cases of insulated boundary conditions are compared to radiation boundary conditions. The incorporation of radiation at the boundary is shown to decrease the operating temperature of the cell where heat loss at the boundaries results in significant temperature differences at the boundaries when compared to the insulated case. However, the current density distribution is not shown to be altered by including radiation heat loss. The performance of the cell is slightly reduced due to the lower operating temperature with radiation heat loss. However, the voltage is only reduced by approximately $2 \mathrm{mV}$ between the insulated and $823 \mathrm{~K}$ cases resulting in similar cell performance.

\section{Conclusion}

The two modeling methodologies for estimating dynamic electrochemical response were shown to be in good agreement with prior modeling efforts where the electro- 
chemical response in the thermal time scale was the basis of comparison. The comparison of the modeling methodology on cell-level dynamics was explored to supplement the steady-state analysis performed in the companion paper by the authors. Modeling comparisons showed small differences in the dynamic variables of interest in both flow configurations. However, the counter-flow configuration has a more pronounced difference particularity in the maximum PEN temperature gradient. The dynamic response of a 'quasi' 2-D model was shown to differ from the 1-D case when capturing the effects of internal methane reforming in the small time scale ( 0.5 seconds) where the dynamics of the fuel species stored in the porous media affect the dynamic electrochemical response of the cell. The small time scale electrochemical dynamic differences are further exaggerated through delays in the fuel flow rate where the fuel stored in the volume of the anode support plays a crucial role in the dynamic response. Since dynamic system-level simulation has primarily identified the anodic fuel depletion as a key problem facing dynamic SOFC operation, capturing the effects of the dynamic fuel species in the anode support layer becomes import in modeling at the system-level. Radiation heat loss at the cell boundaries is also shown to alter the PEN temperature distribution but not the electrochemical performance of the cell.

\section{Acknowledgments}

The authors thank the John Poate Fellowship and the National Science Foundation for partial support of this research effort under award \#CNS-0931748.

\section{References}

[1] E. Achenbach. Three-dimensional and time-dependent simulation of a planar solid oxide fuel cell stack. Journal of Power Sources, 49:333-348, 1994.

[2] P. Aguiar, C.S. Adjiman, and N.P. Brandon. Anode-supported intermediate tem- 
perature direct internal reforming solid oxide fuel cell. I: model-based steadystate performance. Journal of Power Sources, 138:120-136, 2004.

[3] P. Aguiar, C.S. Adjiman, and N.P. Brandon. Anode-supported intermediatetemperature direct internal reforming solid oxide fuel cell II. model-based dynamic performance and control. Journal of Power Sources, 147:136-147, 2005.

[4] Kevin J Albrecht and Robert J Braun. The effect of coupled mass transport and internal reforming on modeling of solid oxide fuel part i: Channel-level model development and steady-state comparison. Journal of Power Sources, Submitted, 2014.

[5] Y. Mollayi Barzi, M. Ghassemi, and M.H. Hamedi. A 2D transient numerical model combining heat/mass transport effects in a tubular solid oxide fuel cell. Journal of Power Sources, 192(1):200-207, 2009.

[6] Debangsu Bhattacharyya, Raghunathan Rengaswamy, and Caine Finnerty. Dynamic modeling and validation studies of a tubular solid oxide fuel cell. Chemical Engineering Science, 64(9):2158-2172, 2009.

[7] A. Chaisantikulwat, C. Diaz-Goano, and E.S. Meadows. Dynamic modelling and control of planar anode-supported solid oxide fuel cell. Computers \& Chemical Engineering, 32(10):2365-2381, 2008.

[8] Andrew M. Colclasure. Dynamic, physically based, models of tublar, solid-oxide fuel cells. Master's thesis, Colorado School of Mines, 2007.

[9] Andrew M. Colclasure, Borhan M. Sanandaji, Tyrone L. Vincent, and Robert J. Kee. Modeling and control of tubular solid-oxide fuel cell systems. i: Physical models and linear model reduction. Journal of Power Sources, 196(1):196-207, 2011. 
[10] D.J. Hall and R.G. Colclaser. Transient modeling and simulation of a tubular solid oxide fuel cell. Energy Conversion, IEEE Transactions on, 14(3):749-753, 1999.

[11] P. Iora, P. Aguiar, C.S. Adjiman, and N.P. Brandon. Comparison of two IT DIRSOFC models: Impact of variable thermodynamic, physical, and flow properties. steady-state and dynamic analysis. Chemical Engineering Science, 60(11):2963$2975,2005$.

[12] Ying-Wei Kang, Jun Li, Guang-Yi Cao, Heng-Yong Tu, Jian Li, and Jie Yang. A reduced 1d dynamic model of a planar direct internal reforming solid oxide fuel cell for system research. Journal of Power Sources, 188(1):170-176, 2009.

[13] P. Kazempoor, V. Dorer, and F. Ommi. Modelling and performance evaluation of solid oxide fuel cell for building integrated co- and polygeneration. Fuel Cells, 10(6):1074-1094, 2010.

[14] Pejman Kazempoor, Fathollah Ommi, and Viktor Dorer. Response of a planar solid oxide fuel cell to step load and inlet flow temperature changes. Journal of Power Sources, 196(21):8948-8954, 2011.

[15] Fabian Mueller, Jacob Brouwer, Faryar Jabbari, and Scott Samuelsen. Dynamic simulation of an integrated solid oxide fuel cell system including current-based fuel flow control. Journal of Fuel Cell Science and Technology, 3:144-154, 2006.

[16] Arata Nakajo, Zacharie Wuillemin, Jan Van herle, and Daniel Favrat. Simulation of thermal stresses in anode-supported solid oxide fuel cell stacks. part I: Probability of failure of the cells. Journal of Power Sources, 193(1):203 - 215, 2009.

[17] J Padullés, G.W Ault, and J.R McDonald. An integrated SOFC plant dynamic 
model for power systems simulation. Journal of Power Sources, 86:495-500, 2000.

[18] M. Peksen. 3d transient multiphysics modelling of a complete high temperature fuel cell system using coupled $\{\mathrm{CFD}\}$ and $\{\mathrm{FEM}\}$. International Journal of Hydrogen Energy, 39(10):5137 - 5147, 2014.

[19] Yutong Qi, Biao Huang, and Karl T. Chuang. Dynamic modeling of solid oxide fuel cell: The effect of diffusion and inherent impedance. Journal of Power Sources, 150(0):32-47, 2005.

[20] Yutong Qi, Biao Huang, and Jingli Luo. Dynamic modeling of a finite volume of solid oxide fuel cell: The effect of transport dynamics. Chemical Engineering Science, 61(18):6057-6076, 2006.

[21] Borhan M. Sanandaji, Tyrone L. Vincent, Andrew M. Colclasure, and Robert J. Kee. Modeling and control of tubular solid-oxide fuel cell systems: Ii. nonlinear model reduction and model predictive control. Journal of Power Sources, 196(1):208-217, 2011.

[22] K. Sedghisigarchi and A. Feliachi. Dynamic and transient analysis of power distribution systems with fuel cells-part I: fuel-cell dynamic model. Energy Conversion, IEEE Transactions on, 19(2):423-428, 2004.

[23] Handa Xi. Dynamic modeling and Control of Planar SOFC Power Systems. PhD thesis, University of Michigan, 2007. 


\section{List of Figures}

1. Dynamic benchmarking to previous channel-level modeling efforts as compiled by Qi et al. [20]

2. Dynamic co-flow cell potential response of the 1-D and 'quasi' 2-D models for step changes in current density

3. Dynamic co-flow maximum PEN temperature response of the 1-D and 'quasi' 2-D models for step changes in current density

4. Dynamic co-flow maximum PEN temperature gradient response of the 1-D and 'quasi' 2-D models for step changes in current density

5. Dynamic counter-flow cell potential response of the 1-D and 'quasi' 2-D models for step changes in current density

6. Dynamic counter-flow maximum PEN temperature response of the 1-D and 'quasi' 2-D models for step changes in current density

7. Dynamic counter-flow maximum PEN temperature gradient response of the 1-D and 'quasi' 2-D models for step changes in current density

8. Dynamic counter-flow 'quasi' 2-D comparison of including and neglecting mass transport dynamic and considering delays in fuel and air flow rates

9. Steady-state comparison of radiation boundary conditions on the model PEN and interconnect temperature profiles 


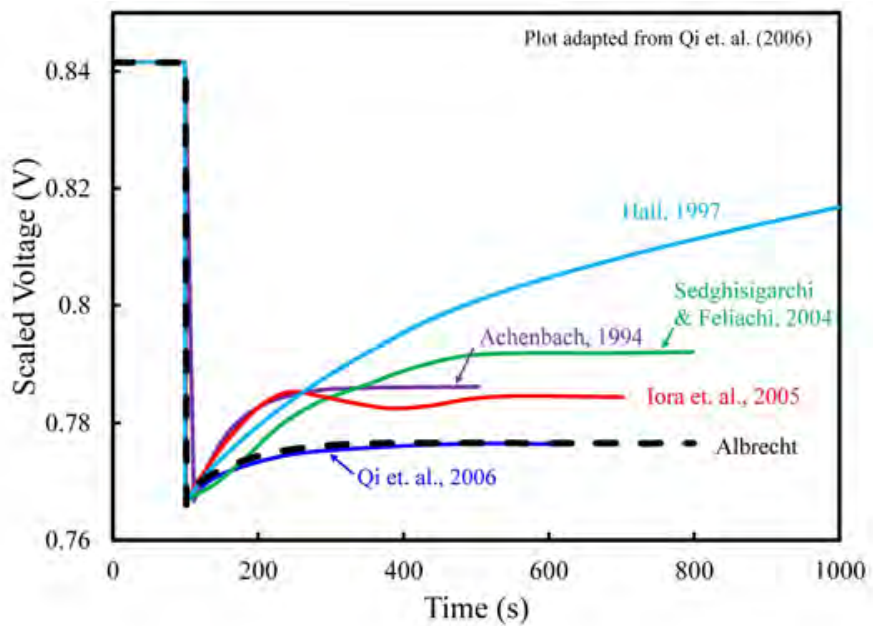

Figure 1: Dynamic benchmarking to previous channel-level modeling efforts as compiled by Qi et al. [20] 


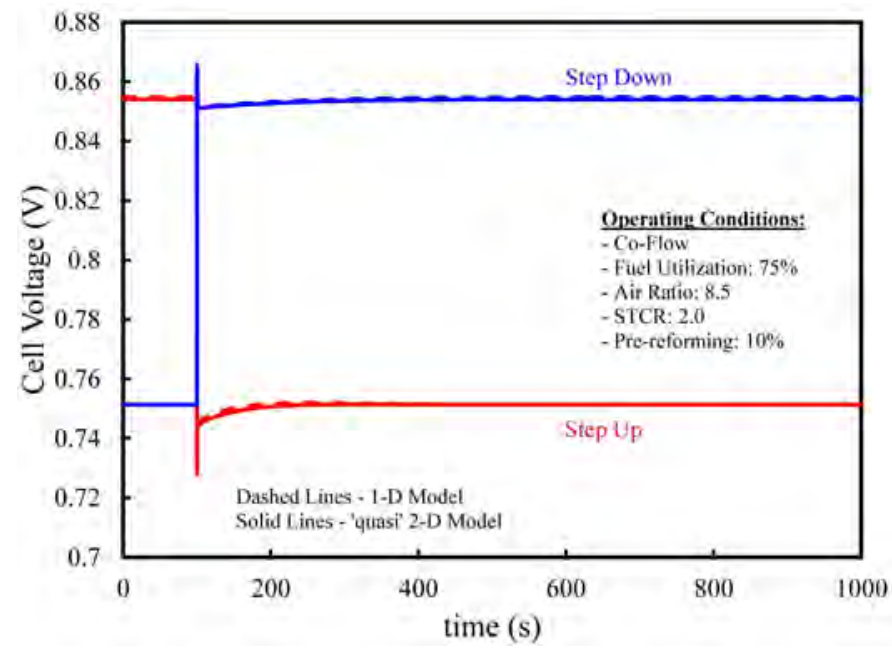

Figure 2: Dynamic co-flow cell potential response of the 1-D and 'quasi' 2-D models for step changes in current density 


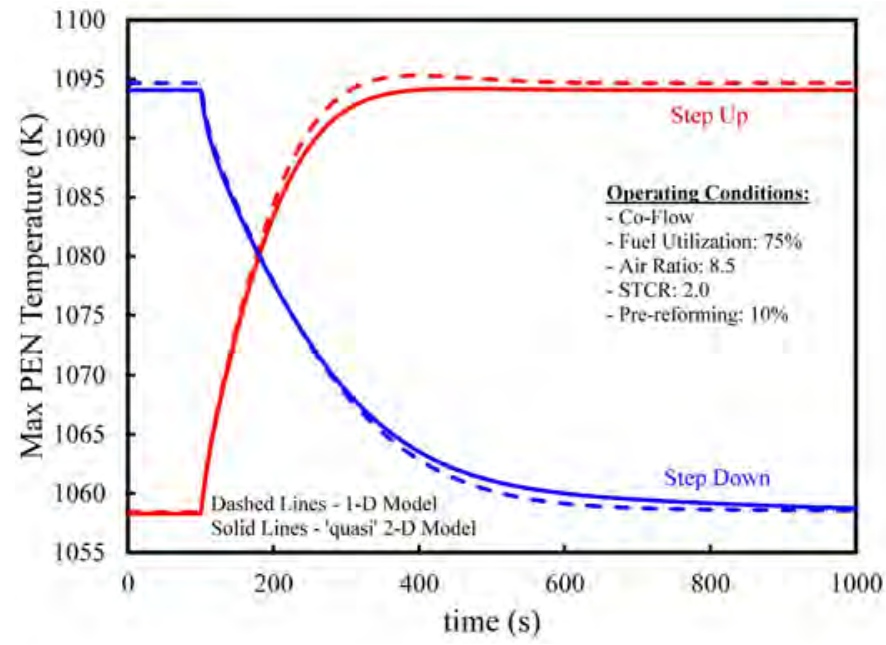

Figure 3: Dynamic co-flow maximum PEN temperature response of the 1-D and 'quasi' 2-D models for step changes in current density 


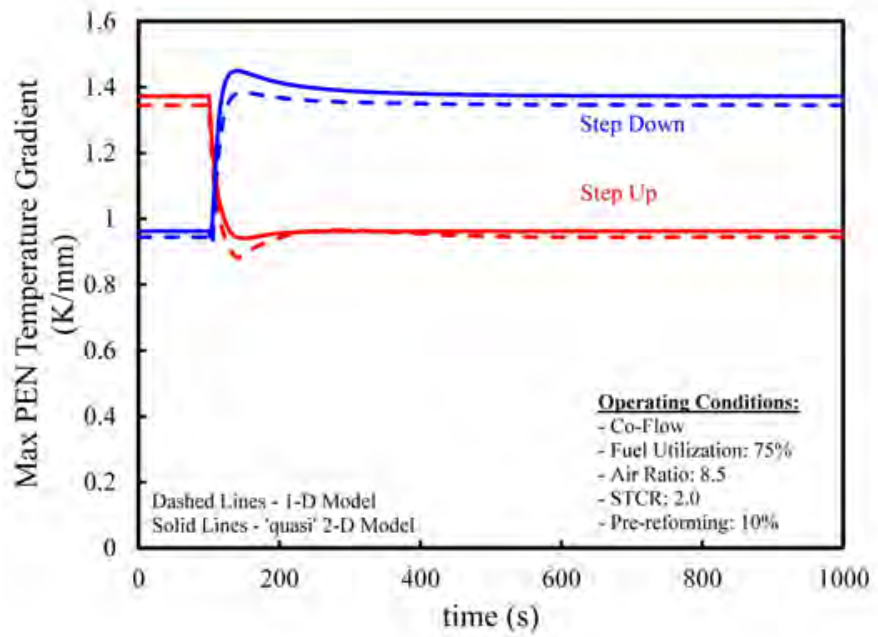

Figure 4: Dynamic co-flow maximum PEN temperature gradient response of the 1-D and 'quasi' 2-D models for step changes in current density 


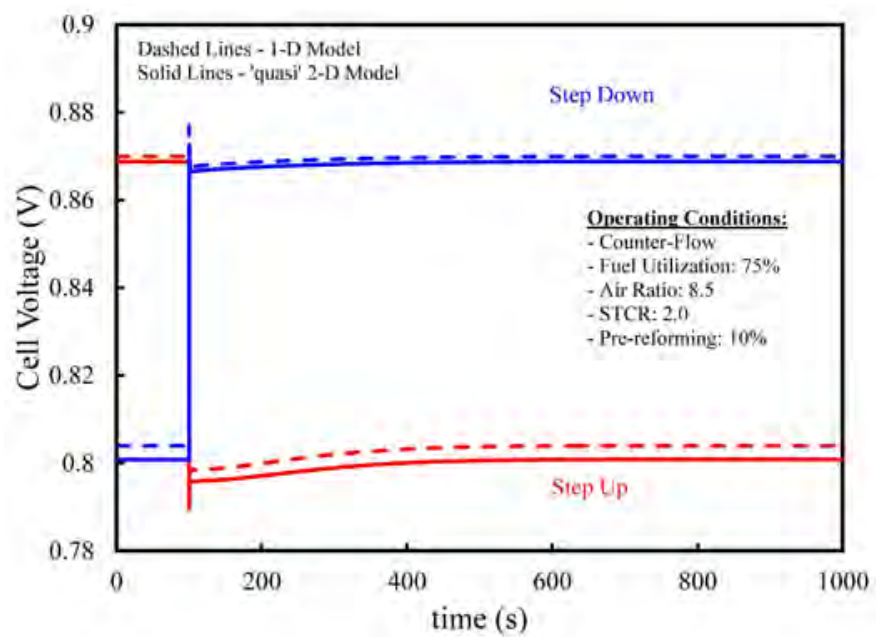

Figure 5: Dynamic counter-flow cell potential response of the 1-D and 'quasi' 2-D models for step changes in current density 


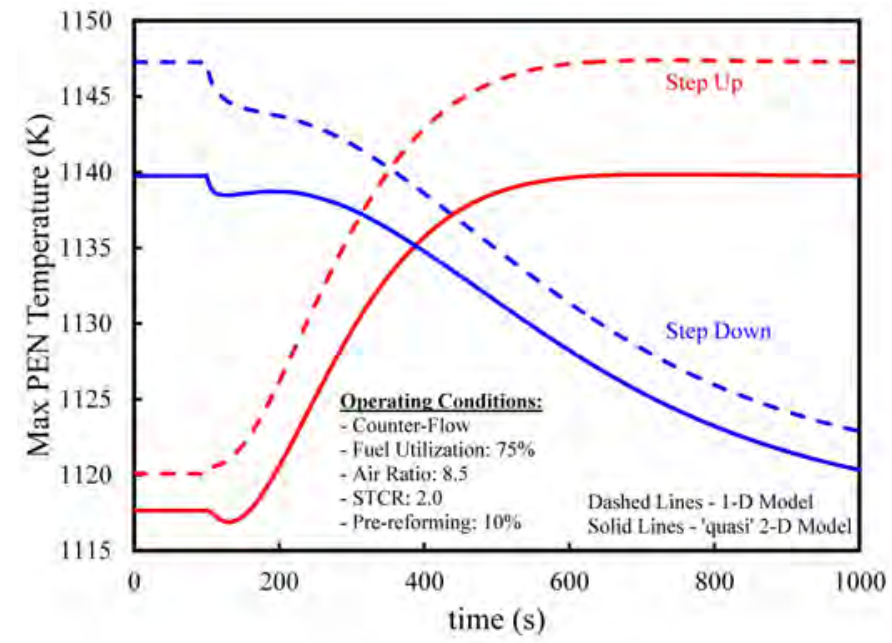

Figure 6: Dynamic counter-flow maximum PEN temperature response of the 1-D and 'quasi' 2-D models for step changes in current density 


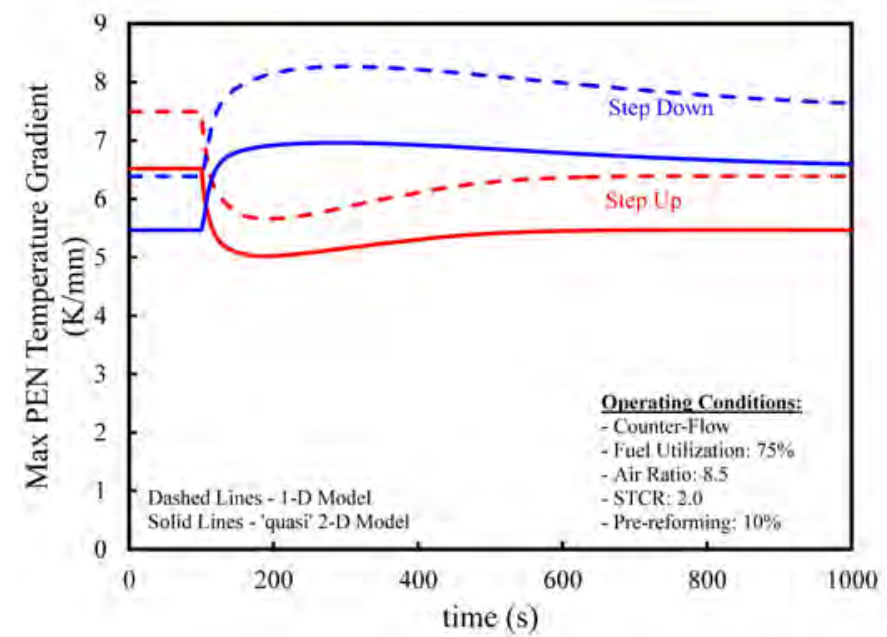

Figure 7: Dynamic counter-flow maximum PEN temperature gradient response of the 1-D and 'quasi' 2-D models for step changes in current density 


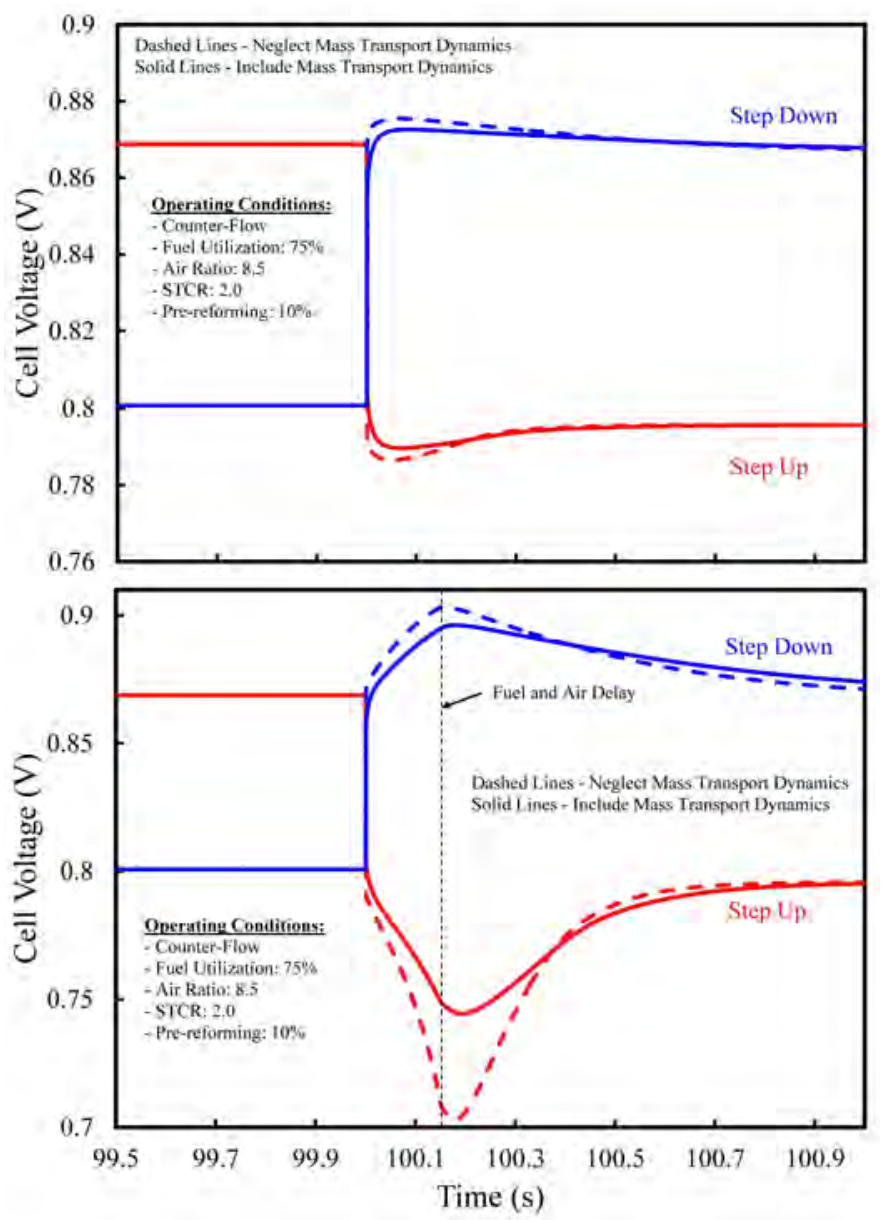

Figure 8: Dynamic counter-flow 'quasi' 2-D comparison of including and neglecting mass transport dynamic and considering delays in fuel and air flow rates 


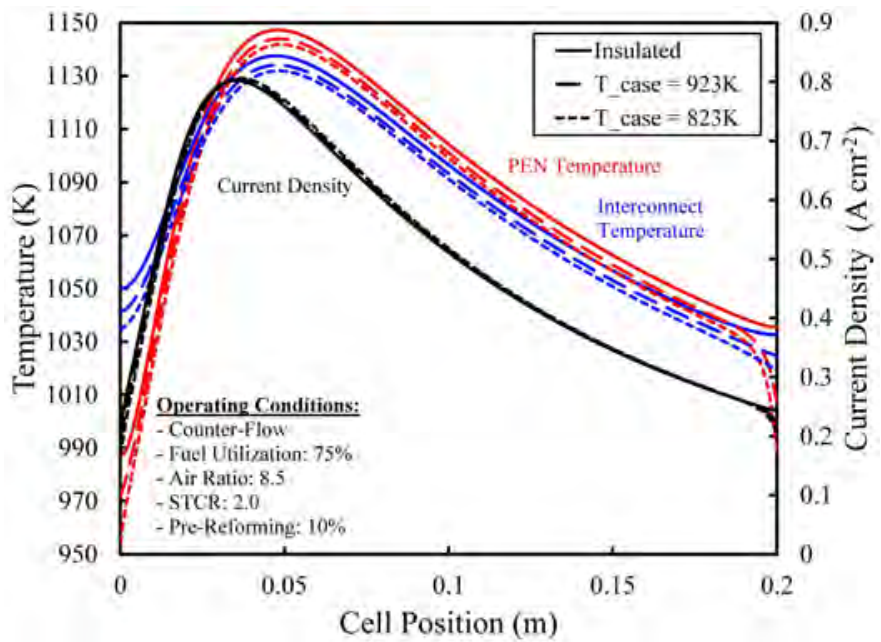

Figure 9: Steady-state comparison of radiation boundary conditions on the model PEN and interconnect temperature profiles 


\section{List of Tables}

1. Comparison of operating conditions for the transient model benchmarking

2. SOFC operating parameters 


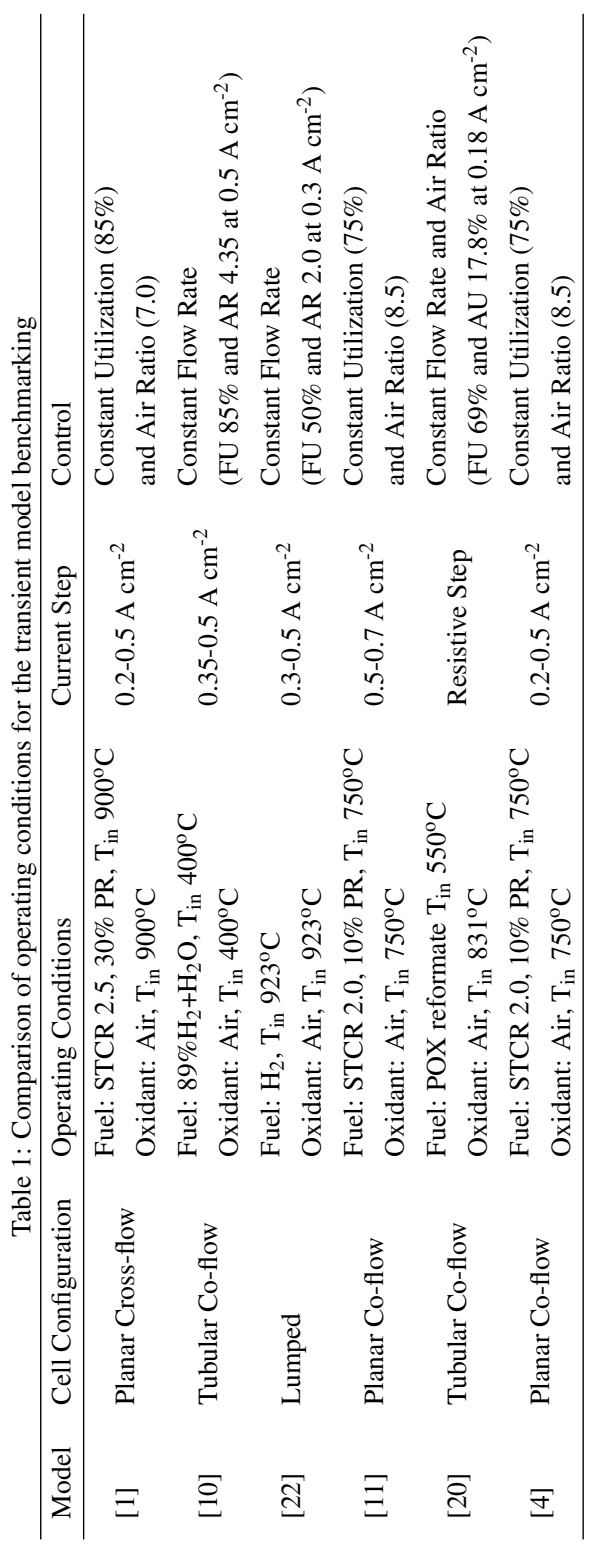


Table 2: SOFC operating parameters

\begin{tabular}{cc}
\hline Operating Parameter & Value \\
\hline Fuel Utilization & $75 \%$ \\
Fuel Composition & $\mathrm{x}_{\mathrm{CH}_{4}}=0.281$ \\
& $\mathrm{x}_{\mathrm{CO}_{2}}=0.0271$ \\
& $\mathrm{x}_{\mathrm{CO}}=0.00415$ \\
& $\mathrm{x}_{\mathrm{H}_{2} \mathrm{O}}=0.566$ \\
& $\mathrm{x}_{\mathrm{H}_{2}}=0.121$ \\
Fuel Temperature & $1023 \mathrm{~K}$ \\
Air Ratio & 8.5 \\
Air Temperature & $1023 \mathrm{~K}$ \\
\hline
\end{tabular}

